\title{
A channel quality indicator (CQI) prediction scheme using feed forward neural network (FF-NN) technique for MU-MIMO LTE system
}

\begin{abstract}
In Multi User-Multiple-in Multiple-Out - Long Term Evolution (MU-MIMO-LTE) networks, Channel Quality indicator (CQI) plays a vital role. CQI is crucial in describing the channel information to assign appropriate modulation and coding scheme (MCS). However, obtaining CQI values for each transmission time interval (TTI) inevitably entails use and can lead to an undesirable degradation in spectral efficiency (SE) as well as increasing the error rate. Therefore, providing an accurate and reliable CQI with low overhead is an intricate task. In this paper, a CQI prediction scheme using Feed Forward-Neural Network (FF-NN) algorithm for MU-MIMO-LTE Advanced systems is proposed. Initially, a channel model for MUMIMO-LTE advanced network is carried out. Through this model, CQI is predicted and the obtained values are compressed using a feedback compression technique. Finally, the proposed technique makes use of FF-NN algorithm to train and achieve enhanced CQI values. Further, an enhanced and accurate CQI values are acquired. Results show that the system SE of single user (SU)-MIMO proportionally increases with the SNR values at the cost of BER. Therefore, a MU-MIMO CQI prediction scheme is recommended to improve the tradeoff between BER and SE.
\end{abstract}

Keyword: CQI feedback; Feed forward neural network technique; LTE; MU-MIMO 